\title{
A novel alternative to the Commando procedure: Constructing a neo-aortic root by anchoring to the sewing ring of the replaced mitral valve
}

\author{
Stefan Elde, MD, Andreas de Biasi, MD, Y. Joseph Woo, MD, and Élan Burton, MD, MHA, Stanford, Calif
}

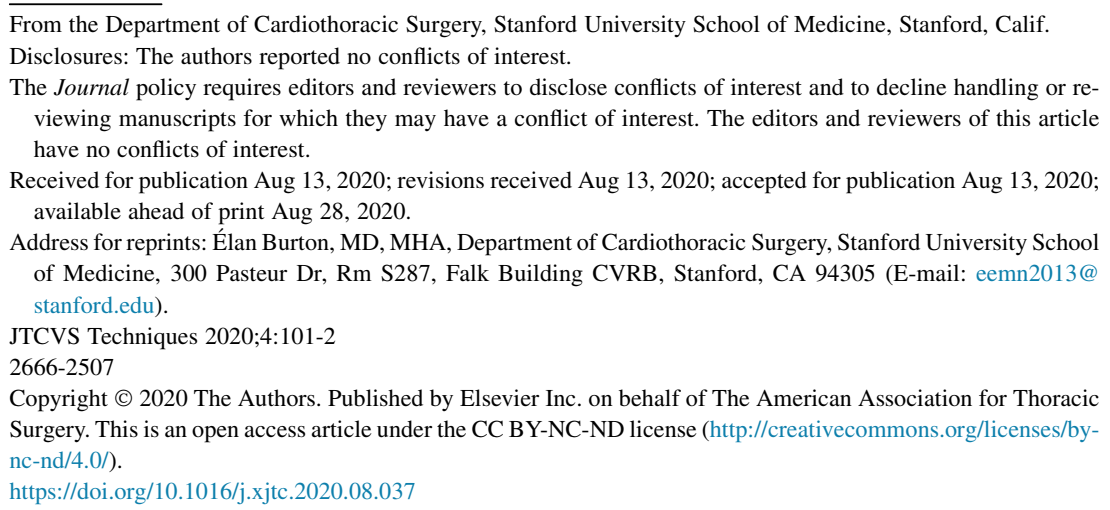

The first aortic and mitral valve replacement via an aortotomy was reported by Carmichael and colleagues ${ }^{1}$ in 1983. David and colleagues used the same exposure in $1997^{2}$ to pioneer what came to be named the Commando procedure, which is to reconstruct the aortomitral curtain to allow double-valve replacement in cases of infective endocarditis. Here, we present a novel iteration of this procedure using the mitral sewing ring as an anchor for the neo-aortic root.

\section{CASE REPORT}

A 58-year-old male patient underwent aortic valve replacement and ascending-hemiarch replacement for concurrent endocarditis and Stanford type A aortic dissection. Four months later, he was readmitted with pleuritic chest pain. Computed tomography angiography of the chest showed contrast extravasation into a perigraft fluid collection surrounding the root and ascending graft. Transthoracic echocardiography showed severe aortic regurgitation and moderate mitral regurgitation suggestive of bioprosthetic endocarditis. He remained afebrile, hemodynamically stable, and his white blood cell count was normal. He was started on intravenous antibiotics and scheduled for urgent surgery 3 days later.

Before the chest was opened, the left axillary artery was dissected and an 8-mm Dacron graft anastomosed for the arterial cardiopulmonary bypass line. Once the chest was entered, cardiopulmonary bypass was initiated in the standard fashion. Upon entering the pseudoaneurysm, we found purulent material that encompassed the root and graft. The root was dehisced beneath the right coronary button and the noncoronary cusp with associated disintegration of the aortomitral curtain and dehiscence of the anterior mitral leaflet

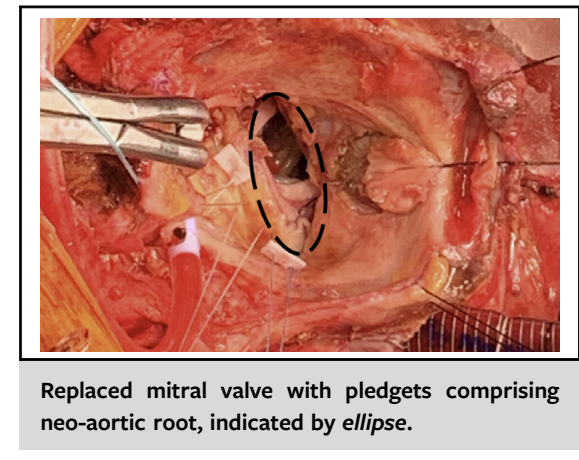

\begin{abstract}
CENTRAL MESSAGE
Here, we report a novel technique for replacement of the mitral and aortic valves via an aortotomy in a patient with recent Stanford type A aortic dissection and multivalve infective endocarditis.
\end{abstract} intact. The native aortic root, previous bioprosthetic aortic valve, and remnant of the anterior mitral leaflet were removed and pledgeted sutures were placed around the mitral annulus. A supra-annular \#33 St Jude Epic biologic mitral valve (St Paul, Minn) was implanted in reverse orientation as previously described, obviating the need to enter the left atrium (Figure 2). ${ }^{3}$ Pledgeted neo-aortic root sutures were placed across the regions of the right and left fibrous trigones just inferior to the territory of the noncoronary cusp and anchored to the segment of the mitral sewing ring that corresponded to the native anterior mitral leaflet. A \#27 Edwards Magna biologic aortic valve (Irvine, Calif) was sewn to a 30-mm HEMASHIELD straight graft (Gothenburg, Sweden) and then into the left ventricular outflow tract (LVOT) and mitral valve sewing ring. The coronary buttons were reimplanted on the straight graft, which was subsequently anastomosed to the remaining distal hemiarch graft from the index operation. 


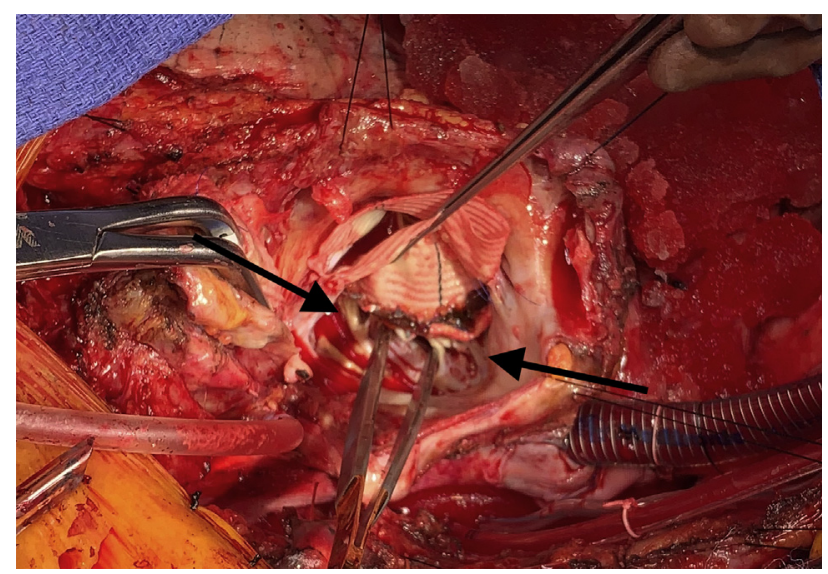

FIGURE 1. Intraoperative view from the patient's right. The area between the 2 arrows show the area of dehiscence of the previous aortic valve conduit from the aortic root, specifically between the right coronary cusp and the noncoronary cusp, as indicated by the vertical forceps at 7 o'clock.

Postcardiopulmonary bypass transesophageal echocardiography showed no aortic or mitral regurgitation.

The patient was discharged to rehabilitation on postoperative day 16 in stable condition with a permanent pacemaker and lifelong antibiotics. Follow-up transthoracic echocardiography at 5 months showed aortic and mitral valve gradients of $7 \mathrm{~mm} \mathrm{Hg}$ and $6.5 \mathrm{~mm} \mathrm{Hg}$, respectively. At this visit, he described continued improvement in his strength and energy levels despite his outpatient physical therapy being interrupted by the coronavirus disease 2019 pandemic. Consent for this case report was provided by the patient as part of our institution's consent for surgery.

\section{DISCUSSION}

Infective endocarditis involving both the aortic and mitral valves is particularly well suited for a transaortic exposure, as opposed to the more traditional left atrial or transseptal approach for mitral procedures. ${ }^{3}$ In contrast to David and colleagues' reconstruction of the intervalvular fibrous body with bovine pericardium or $\mathrm{Dacron}^{2}$ or the hemiCommando developed by the Cleveland Clinic group, ${ }^{4}$ in this report we describe a novel technique for the replacement of the aortic and mitral valves affected by infective endocarditis. Rather than the complex reconstruction of the aortomitral curtain and aortic root, in this case we used part of the sewing ring of the replaced mitral valve in addition to healthy LVOT tissue as an anchor for which the new aortic valve is secured. In this case, the 2 annuli were "hinged" together at the contact point between the sewing ring segment around the bioprosthetic anterior mitral leaflet and the bioprosthetic noncoronary cusp of the aortic valve. Provided that the surgeon is confident that the intertrigonal tissue and mitral annulus is free of inflammatory damage

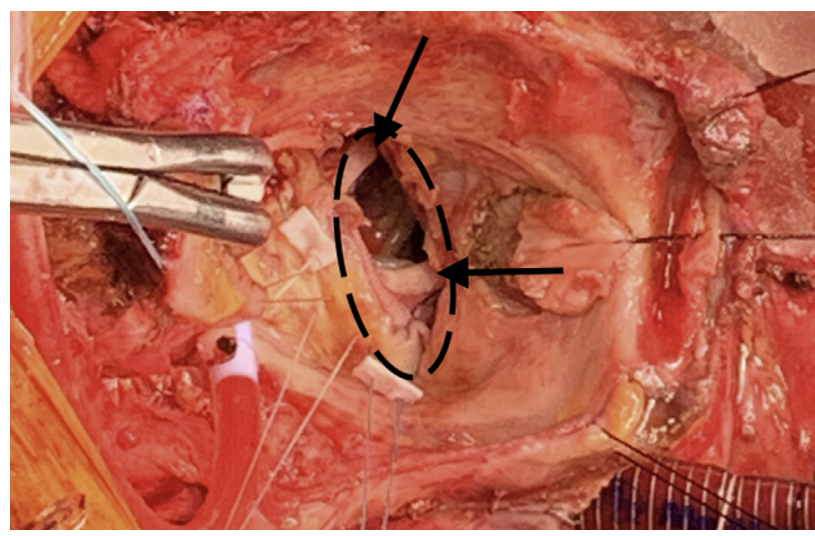

FIGURE 2. Intraoperative view from the patient's right looking down the aortotomy. Two of the struts of the replaced mitral valve can be seen pointing toward 1 and 3 o'clock (arrows), respectively. In this photograph, pledgets are being secured to the mitral annulus, corresponding with the location of the resected native anterior mitral leaflet, which will be used to anchor the neo-aortic root once the tension on the mitral valve is relaxed. The future location of the aortic valve is indicated by the dashed black ellipse.

and retains enough structural integrity to support these anchoring sutures, this technique results in a durable repair without LVOT obstruction. Considering the recent data on graft selection in endocarditis cases have shown similar outcomes between bioprosthetic valves and homografts, ${ }^{5-7}$ due to the urgent setting of this procedure and the mitral valve requiring replacement, we elected a bioprosthetic aortic valve instead of an aortic homograft. The advantage of this technique is that it simplified the approach to the common problem of multivalvular infective endocarditis and may spare critically ill patients the morbidities and mortality risk associated with a prolonged cardiopulmonary bypass run.

\section{References}

1. Carmichael MJ, Cooley DA, Favor AS. Aortic and mitral valve replacement through a single transverse aortotomy: a useful approach in difficult mitral valve exposure. Tex Heart Inst J. 1983;10:415-9.

2. David TE, Kuo J, Armstrong S. Aortic and mitral valve replacement with reconstruction of the intervalvular fibrous body. J Thorac Cardiovasc Surg. 1997;114: 766-72.

3. Frederick JR, Woo YJ. Transaortic mitral valve replacement. Ann Thorac Surg. 2012;94:302-4.

4. Navia J, Al-Ruzzeh S, Gordon S, Fraser T, Agüero O, Rodríguez L. The incorporated aortomitral homograft: a new surgical option for double valve endocarditis. J Thorac Cardiovasc Surg. 2010;139:1077-81.

5. Jassar AS, Bavaria JE, Szeto WY, Moeller PJ, Maniaci J, Milewski RK, et al. Graft selection for aortic root replacement in complex active endocarditis: does it matter? Ann Thorac Surg. 2012;93:480-7.

6. Avierinos J-F, Thuny F, Chalvignac V, Giorgi R, Tafanelli L, Casalta JP, et al. Surgical treatment of active aortic endocarditis: homografts are not the cornerstone of outcome. Ann Thorac Surg. 2007;84:1935-42.

7. Kim JB, Ejiofor JI, Yammine M, Camuso JM, Walsh CW, Ando M, et al. Are homografts superior to conventional prosthetic valves in the setting of infective endocarditis involving the aortic valve? J Thorac Cardiovasc Surg. 2016;151: 1239-48.e2. 